\title{
The Correspondence between Fluid Balance and Body Weight Change Measurements in Critically Ill Adult Patients
}

\author{
Ana Carolina Peçanha Antonio1*, Vivian Rodrigues Fernandes², Karina de Oliveira Azzolin³ \\ 1 Hospital de Clínicas de Porto Alegre, Porto Alegre, Brazil \\ 2 Fellow nurse, Instituto de Cardiologia, Porto Alegre, Brazil \\ 3 Universidade Federal do Rio Grande do Sul Instituto de Ciencias Basicas da Saude, Porto Alegre, Brazil
}

\begin{abstract}
Introduction: Positive fluid status has been associated with a worse prognosis in intensive care unit (ICU) patients. Given the potential for errors in the calculation of fluid balance totals and the problem of accounting for indiscernible fluid losses, measurement of body weight change is an alternative non-invasive method commonly used for estimating body fluid status. The objective of the study is to compare the measurements of fluid balance and body weight changes over time and to assess their association with ICU mortality. Methods: This prospective observational study was conducted in the 34-bed multidisciplinary ICU of a tertiary teaching hospital in southern Brazil. Adult patients were eligible if their expected length of stay was more than 48 hours, and if they were not receiving an oral diet. Clinical demographic data, daily and cumulative fluid balance with and without indiscernible water loss, and daily and total body weight changes were recorded. Agreement between daily fluid balance and body weight change, and between cumulative fluid balance and total body weight change were calculated. Results: Cumulative fluid balance and total body weight change differed significantly among survivors and non survivors respectively, $+2.53 \mathrm{~L}$ versus $+5.6 \mathrm{~L}(\mathrm{p}=0.012)$ and $-3.05 \mathrm{~kg}$ vs $-1.1 \mathrm{~kg}(p=0.008)$. The average daily difference between measured fluid balance and body weight was $+0.864 \mathrm{~L} / \mathrm{kg}$ with a wide interval: -3.156 to $+4.885 \mathrm{~L} / \mathrm{kg}$, which remained so even after adjustment for indiscernible losses (mean bias: +0.288 ; limits of agreement between -3.876 and $+4.452 \mathrm{~L} / \mathrm{kg}$ ). Areas under ROC curve for cumulative fluid balance, cumulative fluid balance with indiscernible losses and total body weight change were, respectively, $0.65,0.56$ and $0.65(p=0.14)$. Conclusion: The results indicated the absence of correspondence between fluid balance and body weight change, with a more significant discrepancy between cumulative fluid balance and total body weight change. Both fluid balance and body weight changes were significantly different among survivors and non-survivors, but neither measurement discriminated ICU mortality.
\end{abstract}

Keywords: fluid balance, body weight change, critical illness

Received: 23 June 2020 / Accepted: 8 December 2020

\section{INTRODUCTION}

Positive fluid status has been consistently associated with worse prognosis in critically ill adult patients [1-8]. Daily and cumulative fluid balance are routine nursing activities in an intensive care unit (ICU), and are widely used as a basis for decisions concerning other therapies such as the use of diuretics, intravenous fluid administration, and renal replacement therapy. Despite its widespread utilization, the allegedly simple method of subtracting fluid output from the patient's input requires the recording of multiple infusions of fluid such as nutrition, blood products and intravenous medications, as well as hourly urinary output and loss of other body fluids, such as fluid lost from surgical drains. As a result, the process of charting fluid balance is very time-consuming and subject to calculation errors [9], which tend to build up when it comes to cumulative fluid balance [10]. Moreover, accounting for indiscernible water loss or diarrhoea is also inaccurate [11].

Beds with built-in weighing scales can offer an option for the estimation of fluid status changes. One kilogram change in body weight is assumed to equate to one litre gain or loss in body fluid volume [12]. Discipline is also required during the weighing process. Scales need to be zeroed before patients are weighed, and extra items placed on the bed often cannot be removed. Poor compliance in obtaining regular body weight measurements is also an issue which can raise concerns regarding the clinical practicality and costeffectiveness of weighting beds in the management of ICU patients $[13,14]$. 
Previous studies $[8,10,13-17]$ have established that charting fluid balance totals, both under- and overestimated body weight changes in unpredictable patterns. However, short stays in an ICU, enrolment of a specific low-risk population, use of multiple types of scales and emphasis on cumulative fluid balance, prevented generalization of data. No study attempted to assess the superiority of fluid balance or body weight measurements on the prediction of ICU mortality.

The study aimed to compare the correspondence of daily and cumulative fluid balances with simultaneous changes in body weight in critically ill patients admitted to a multidisciplinary ICU.

The null hypothesis was that there was no correspondence between fluid-balance and body weight change measurements in critically-ill adult patients.

\section{MATERIAL AND METHODS}

The study was conducted in the 34-bed multidisciplinary ICU of Hospital de Clínicas de Porto Alegre, a tertiary teaching hospital in southern Brazil.

The local research ethic board approved the methodology, and the committee waived the need for informed consent. The study was registered as NCT04434079 at ClinicalTrials.gov.

The inclusion criteria were adult patients over 18 years of age consecutively admitted to the ICU from June 26th to October 31st 2018, if their expected length of stay was more than 48 hours.

The exclusion criteria were body weight above the bed safe working load of 226kgs (or 500lb) [18], any food taken orally, and incomplete recording of fluid balance or body weight measurements.

All patients were placed on Hill-Rom ${ }^{\text {TM }}$ Total Care bed systems (Batesville, Ind). As stated by the manufacturer [18], the accuracy of the scale is $1 \%$ of the patient's weight, and the repeatability goes from $\pm 0.1 \%$ to $\pm 0.3 \%$. Daily fluid balance of all enteral and parenteral provisions and excretions was performed manually at midnight for each patient and routinely recorded on report sheets by the responsible nurse. Fluid losses were calculated according to the predetermined formula: $800+20 \% \times 800 \times$ (maximum body temperature -37 ; divided by 2 if the patient was intubated), in millilitres. [11]. Weight changes of the soaked linen estimated the output from watery diarrhoea. The cumulative fluid balance was the sum of the daily fluid balance between the day of ICU admission and the day of discharge. To identify arithmetic errors, all ICU fluid balance chart input and output measurements were recalculated. The weighing procedure was obtained at midnight to coincide with the 24-hour fluid balance calculation [18].

Demographic data including age, gender, body mass index, the reason for ICU admission, the severity of illness at the time of ICU admission, comorbidities, caloric and protein intake, the need of diuretics or renal replacement therapy, duration of invasive mechanical ventilation, length of ICU stay, and ICU mortality were recorded.

Descriptive statistics are presented as mean (SD) or median and interquartile range. The Student $t$-test or the Mann-Whitney $U$ test were used to compare continuous variables, and the Chi-square test or the Fisher exact test to compare proportions. The significance level was set at $\alpha=0.05$.

Correlation tests were performed for a relationship between fluid balance and body weight changes. A Bland-Altman assessment for agreement between daily fluid balance and body weight changes and between cumulative fluid balance and total body weight change was calculated. The range of agreement was defined as the mean bias \pm 2 SDs. A receiver operating characteristic (ROC) curve was plotted for each technique according to ICU survival.

Data were analysed using STATA version 16.1 (StataCorp LP, College Station, TX, USA).

\section{RESULTS}

During the study period, there were 469 admissions to the ICU. Of those, ninety-four (20\%) patients were enrolled in the study (Figure 1). Two patients were readmitted, and thirty (31.9\%) died in ICU during the study period. Their baseline characteristics are described in Table 1.

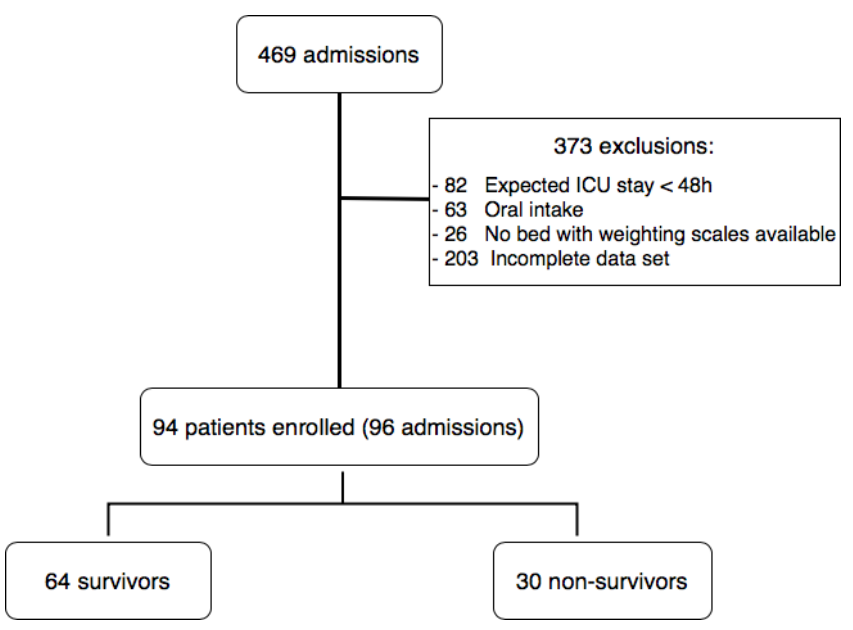

Fig. 1. Study flow. 
The main cause of ICU admission was respiratory sepsis (30.8\%), and ninety (95.7\%) individuals required invasive mechanical ventilation at some point during their ICU stay.
Non-survivors were more likely to receive RRT (53.3\% vs $25 \%$, Chi-square test $\mathrm{p}=0.01$ ) and to suffer from nosocomial infections $(43.3 \%$ vs $18.7 \%$, Chi-square test $\mathrm{p}=0.023)$, and spent more days on mechanical ventilation ( 8 days vs 6 days, Mann-Whitney $U$ test $\mathrm{p}=0.012$ ).

Table 1. Characteristics of the study cohort

\begin{tabular}{|c|c|c|c|c|}
\hline & $\begin{array}{l}\text { All patients } \\
(n=94)\end{array}$ & $\begin{array}{l}\text { Survivors } \\
(n=64)\end{array}$ & $\begin{array}{l}\text { Non-survivors } \\
(n=30)\end{array}$ & p Value \\
\hline Age; years (SD) I & $57.36(17.20)$ & $57.83(17.92)$ & $56.37(15.78)$ & 0.70 \\
\hline Female gender (\%) & $48(51.06)$ & $32(50)$ & $16(53.3)$ & 0.83 \\
\hline SAPS3; points (SD) & $69.03(18.46)$ & $67.78(16.17)$ & $71.67(22.60)$ & 0.34 \\
\hline BMI; kg/m2 (SD) & $27.81(7.25)$ & $28.54(7.49)$ & $26.26(6.54)$ & 0.16 \\
\hline \multicolumn{5}{|l|}{ Origin (\%) } \\
\hline Emergency room & $47(50)$ & $34(53.12)$ & $13(43.33)$ & \multirow{3}{*}{0.35} \\
\hline Hospital ward & $34(36.17)$ & $20(31.25)$ & $14(46.67)$ & \\
\hline Other hospital & $13(13.83)$ & $10(15.62)$ & $3(10)$ & \\
\hline Energy delivered on 3rd day of ICU stay; kcal/day (SD) & $21.05(6.47)$ & $22.12(6.42)$ & $18.50(5.98)$ & 0.014 \\
\hline Protein delivered on 3rd day of ICU stay; g/kg (SD) & $1.42(0.52)$ & $1.49(0.44)$ & $1.25(0.65)$ & 0.045 \\
\hline \multicolumn{5}{|l|}{ Comorbidities (\%) } \\
\hline COPD & $13(13.83)$ & $9(14.06)$ & $4(13.33)$ & 1.0 \\
\hline$E F<35 \%$ & $11(11.70)$ & $8(12.50)$ & $3(10)$ & 1.0 \\
\hline Cirrhosis & $9(9.57)$ & $6(9.38)$ & $3(10)$ & 1.0 \\
\hline Cancer & $23(24.47)$ & $16(25)$ & $7(23.33)$ & 1.0 \\
\hline End-stage renal disease & $7(7.45)$ & $4(6.25)$ & $3(10)$ & 0.68 \\
\hline Immunosuppression & $15(15.96)$ & $8(12.50)$ & $7(23.33)$ & 0.23 \\
\hline \multicolumn{5}{|l|}{ Reason for ICU admission (\%) } \\
\hline Respiratory sepsis & $29(30.85)$ & $18(28.12)$ & $11(36.67)$ & \multirow{8}{*}{0.13} \\
\hline Non respiratory sepsis & $7(7.45)$ & $3(4.69)$ & $4(13.33)$ & \\
\hline Cardiovascular disease & $5(5.32)$ & $5(7.81)$ & $0(0)$ & \\
\hline Coma & $18(19.15)$ & $11(17.19)$ & $7(23.33)$ & \\
\hline Postoperative ARF & $7(7.45)$ & $4(6.25)$ & $3(10)$ & \\
\hline COPD/Asthma & $3(3.19)$ & $2(3.12)$ & $1(3.33)$ & \\
\hline Pulmonary Embolism & $2(2.13)$ & $1(1.56)$ & $1(3.33)$ & \\
\hline ARDS & $23(24.47)$ & $20(31.25)$ & $3(10)$ & \\
\hline \multicolumn{5}{|l|}{ Organ dysfunction at ICU admission* } \\
\hline Hemodynamic & $74(78.72)$ & $50(78.12)$ & $24(80)$ & 1.0 \\
\hline Lung & $86(91.49)$ & $59(92.19)$ & $27(90)$ & 0.71 \\
\hline Renal & $35(37.23)$ & $24(37.5)$ & $11(36.67)$ & 1.00 \\
\hline Hematologic & $25(26.60)$ & $13(20.31)$ & $12(40)$ & 0.078 \\
\hline Liver & $8(8.33)$ & $3(4.55)$ & $5(16.67)$ & 0.10 \\
\hline Neurological & $39(41.49)$ & $23(35.94)$ & $16(53.33)$ & 0.12 \\
\hline Diuretic provision during ICU stay $(\%)^{* *}$ & $30(31.91)$ & $20(31.25)$ & $10(33.33)$ & 1.0 \\
\hline Need of RRT during ICU stay (\%)** & $32(34.04)$ & $16(25)$ & $16(53.33)$ & 0.010 \\
\hline ICU-acquired infection (\%)** & $25(26.60)$ & $12(18.75)$ & $13(43.33)$ & 0.023 \\
\hline MV duration (days)** & $7(3-10)$ & $6(2-9)$ & $8(6-13)$ & 0.012 \\
\hline Length of ICU stay (days)** & $9(6-15)$ & $9.5(6-15.5)$ & $8(6-14)$ & 0.87 \\
\hline Length of hospital stay (days)** & $16(9-27)$ & $16(9.5-28)$ & $15.5(8-27)$ & 0.95 \\
\hline Time between hospitalization and ICU admission (days) & $3(1-10)$ & $3(1-9)$ & $3(1-10)$ & 0.98 \\
\hline
\end{tabular}


Table 2 displays values of fluid balance with and without indiscernible water loss and body weight changes according to ICU survival. The median net body weight change was negative in both survivors and non-survivors, although there was a significantly different between them (respectively $-3.05 \mathrm{~kg}$ vs $-1.1 \mathrm{~kg}$, Mann-Whitney U test $\mathrm{p}=0.008$ ). Only crude cumulative fluid balance, without indiscernible water loss, dif- fered significantly between groups. (Mann-Whitney $U$ test $\mathrm{p}<0.05)$

Altogether, 781 pairs of data were available for analysis between daily fluid balance and body weight changes. As showed by the Bland Altman plot (Figure 2 ), the average daily difference was +0.864 (CI +0.722 to +1.007$)$, but the interval was broad: -3.156 to +4.885 $\mathrm{L} / \mathrm{kg}(\mathrm{A})$.

Table 2. Comparison of fluid balance and weight change of survivors and non-survivors of ICU hospitalization

\begin{tabular}{|c|c|c|c|c|}
\hline & All patients* $(n=94)$ & Survivors $(n=64)$ & Non-survivors $(n=30)$ & p Value \\
\hline First 24h-fluid balance (L) & $+0.95([+0.09]-[+1.78])$ & $+0.84([+0.08]-[+1.6])$ & $+1.26([+0.41]-[+0.27])$ & 0.10 \\
\hline $\begin{array}{l}\text { First } 24 \text { h-fluid balance }+ \\
\text { indiscernible water loss (L) }\end{array}$ & $+0.05([-0.79]-[+0.80])$ & $-0.004([-0.88]-[+0.74])$ & $+0.64([-0.48]-[+1.77])$ & 0.075 \\
\hline First 24h-weight change $(\mathrm{kg})$ & $0.0([-0.9]-[+1.1])$ & $+0.25([-1.45]-[+0.6])$ & $+0.9([-0.1]-[+2.2])$ & $<0.001$ \\
\hline Cumulative fluid balance (L) & $+3.61([-0.11]-[+7.43])$ & $+2.53([-0.4]-[+6.0])$ & $+5.6([+1.85]-[+9.21])$ & 0.012 \\
\hline $\begin{array}{l}\text { Cumulative fluid balance + } \\
\text { indiscernible water loss (L) }\end{array}$ & $-3.89([-7.27]-[-0.1])$ & $-3.91([-7.78]-[-1.1])$ & $-3.33([-6.87]-[+0.97])$ & 0.37 \\
\hline Total weight change (kg) & $-2.55([-4.8]-[+0.1])$ & $-3.05([-6.5]-[-0.7])$ & $-1.1([-3.1]-[+0.9])$ & 0.008 \\
\hline
\end{tabular}

Data are presented as median (interquartile range). *Excluding data from two ICU readmissions
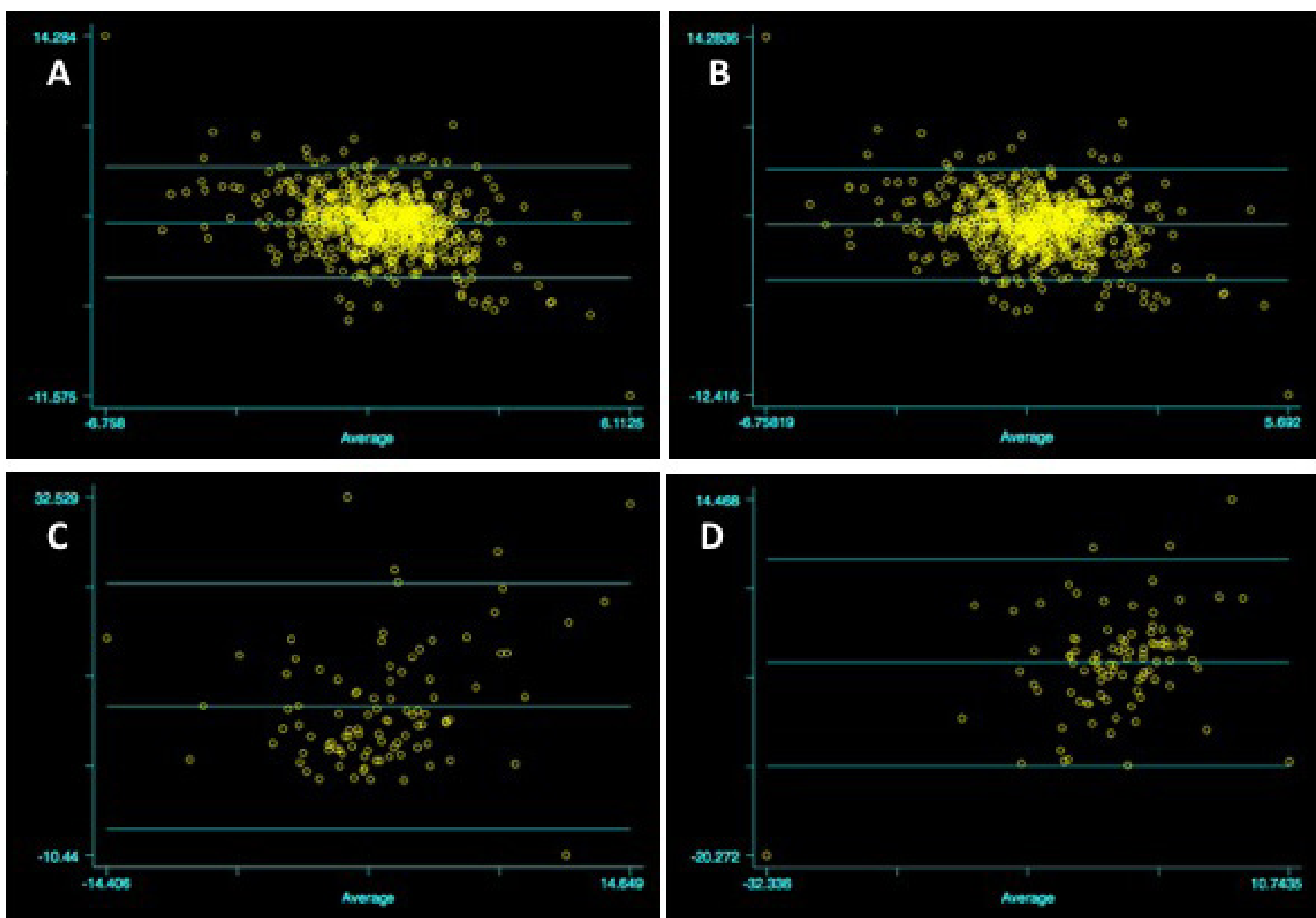

Fig. 2. Bland Altman plot for changes in fluid balance and simultaneous body weight (A) Daily fluid balance and body weight changes. B) Daily fluid balance with indiscernible water loss and body weight changes. C) Cumulative FB and total body weight change. D) Cumulative fluid balance with indiscernible water loss and total body weight change. $X$-axis, mean values for fluid balance and body weight. Y- axis, difference between fluid balance and body weight. 
After adjustment for indiscernible water loss, the mean bias resulted in +0.288 ( $\mathrm{CI}+0.140$ to +0.436 ) $\mathrm{L} / \mathrm{kg}$, limits of agreement $(-3.876$ to $+4.452 \mathrm{~L} / \mathrm{kg}) \mathrm{re}-$ mained wide (B).

The assessment of agreement between cumulative fluid balance and total body weight change occurred in 96 pairs of data, yielding a mean bias of +7.410 (CI +5.917 to +8.902$) \mathrm{L} / \mathrm{kg}$ and an interval of -7.321 to $+22.141 \mathrm{~L} / \mathrm{kg}$ (C). After accounting for indiscernible water loss, the mean difference was -1.463 (CI -2.485 to $-0.441) \mathrm{L} / \mathrm{kg}$, and the interval was -11.552 to $+8.626 \mathrm{~L} /$ $\mathrm{kg}(\mathrm{D})$. The null hypothesis was upheld.

As illustrated in Figure 3, the correlation between daily fluid balance and body weight change was poor (A), even after correction for indiscernible water loss (B). The correlation between cumulative fluid balance and total body weight change went from poor (C) to moderate when indiscernible water loss was included (D).

The areas under the ROC curve showed a failed to poor accuracy for discrimination between ICU survivors and non-survivors. Nor did they significantly differ among cumulative fluid balance, cumulative fluid balance with indiscernible water loss and total weight change (Chi-square test $\mathrm{p}=0.14$ ) (Figure 4$)$.

\section{Discussion}

The results of the study indicate an absence of correspondence between fluid balance and body weight changes, even when daily and systematic measurements are made. There was a large and diffuse dispersion of values, with a more significant discrepancy between cumulative fluid balance and total body weight change.
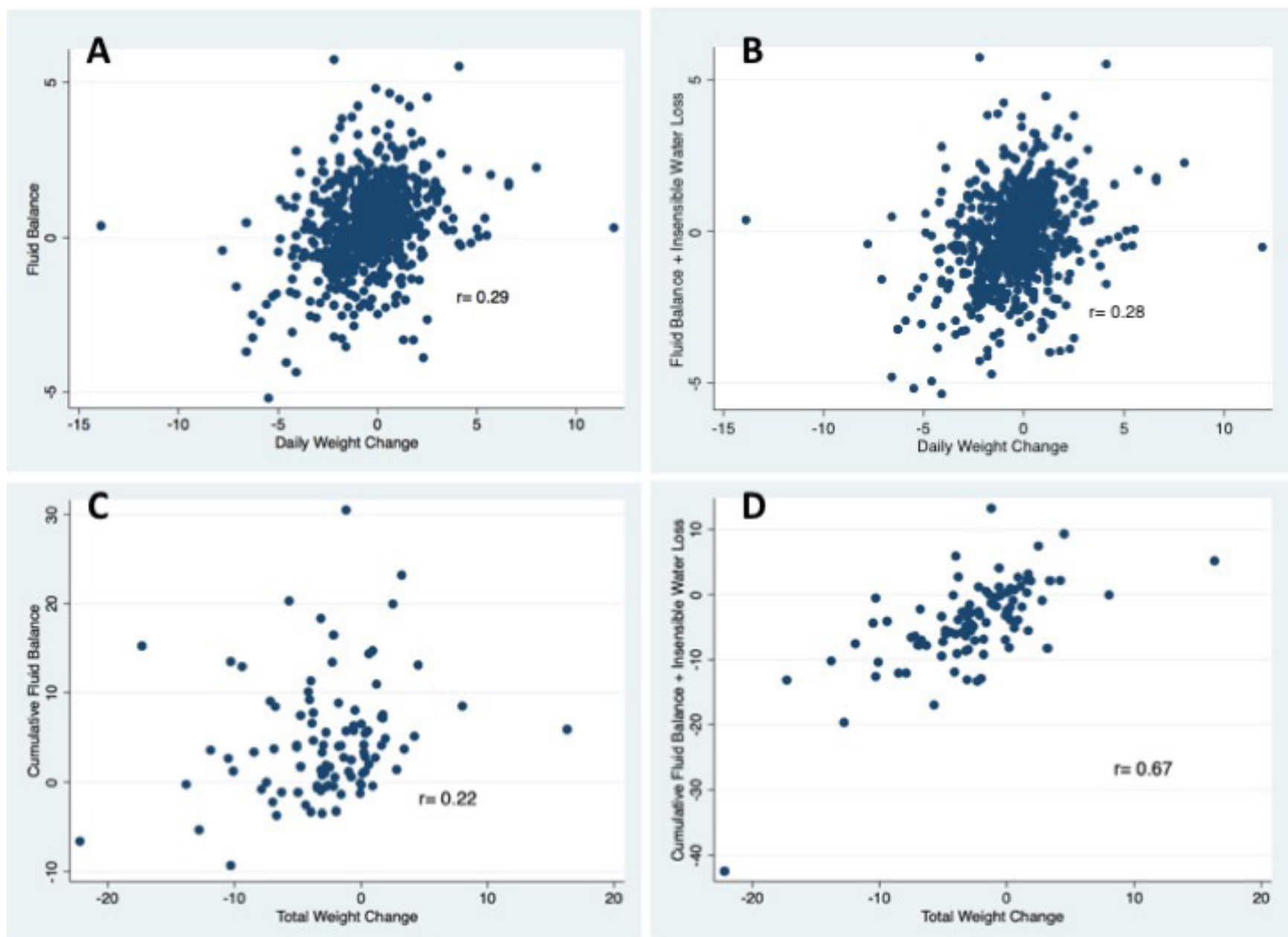

Fig. 3. Correlation between A) daily fluid balance and body weight changes (Spearman rank correlation; $p<0.001)$, B) daily fluid balance with indiscernible water loss and body weight changes (Spearman rank correlation; $p<0.001$ ), C) cumulative fluid balance and total body weight change (Spearman rank correlation; $p=0.03$ ), and D) cumulative fluid balance with indiscernible water loss and total body weight change (Spearman rank correlation; $p<0.001$ ). 


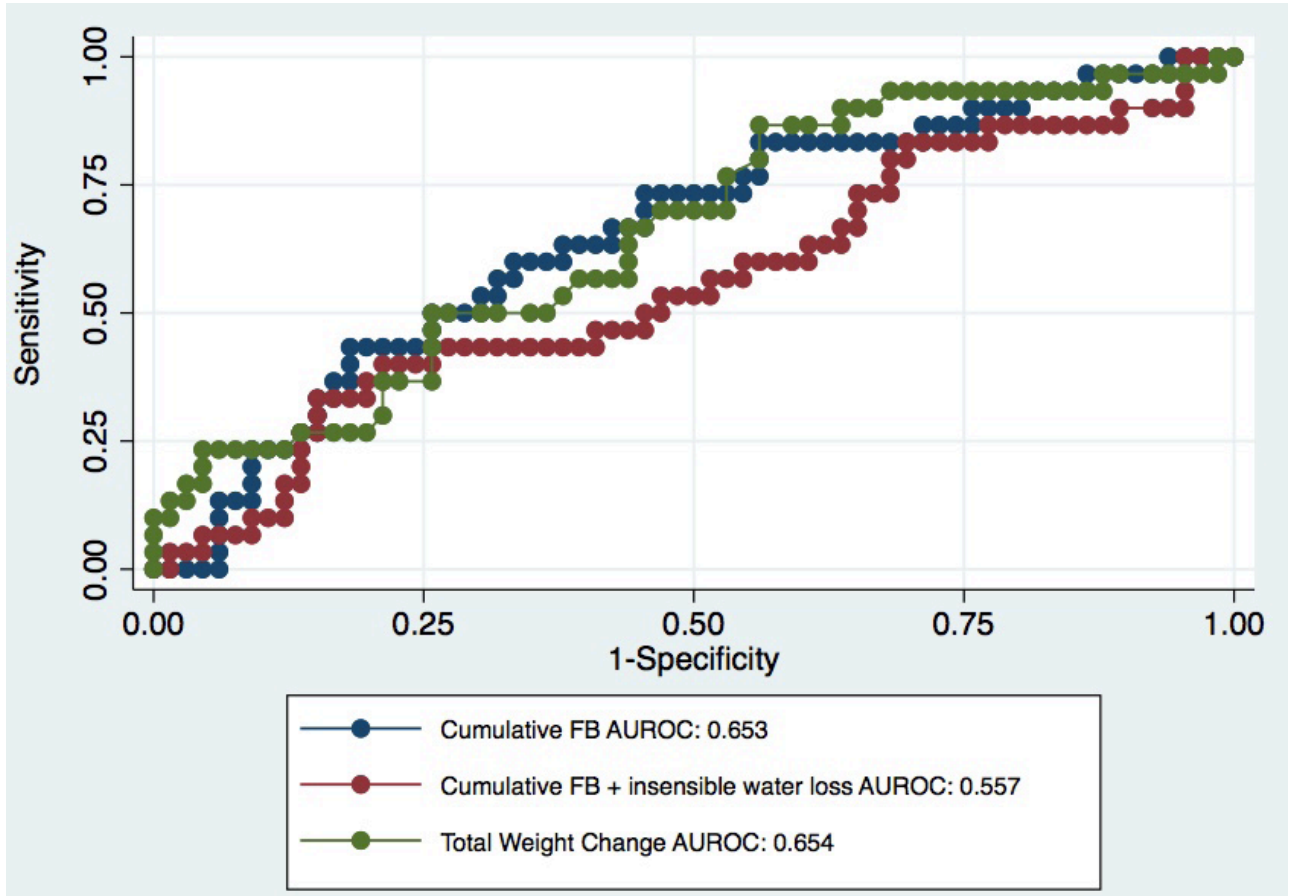

Fig. 4. Areas under ROC curve showing discrimination power of cumulative fluid balance, cumulative fluid balance corrected for indiscernible water loss and total body weight change in predicting ICU mortality.

They correlated with each other, but this had no clinical significance. Neither total change in body weight nor crude cumulative fluid balance provided even moderate discriminatory performance in predicting ICU mortality, although both significantly differed between survivors and non-survivors. To our knowledge, this is the first report encompassing a comprehensive analysis in a general tertiary ICU with a highly heterogeneous cohort and in which the weighting process was entirely performed on integrated bed scales.

Previous studies have also demonstrated poor reliability of fluid balance measures. Moreover, the correlation with body weight changes did not improve when a correction for indiscernible water loss was made [8, $10,13-17]$. Some investigations only focused on net changes $[10,15-17]$ which are subjected to error accumulation, the higher the duration of ICU hospitalization [16]. As there is no consensus in the literature regarding indiscernible water losses, data were presented with and without correction.

Research on daily acquired data confirmed a lack of agreement in specific populations [14, 19] and during short lengths of stay $[8,13]$. One report [19] used only a correlation coefficient and a regression technique which can be misleading as they evaluate only the lin- ear association of two sets of observations. The Bland Altman method provides an adequate analysis of the agreement of data [20]. Moreover, most prior studies implied that body weight change was the gold-standard to be pursued. However, the weighing process needs to be performed in a very standardized fashion in order to be exempt from errors [14].

The body weight obtained by weight-enabled beds cannot be used as a substitute for fluid balance recording or vice versa, as suggested by the weak correlation between them daily. The low accuracy of all net measurements in predicting ICU mortality precludes a conclusion regarding "the best one" to be employed clinically. Indeed, the magnitude of errors associated with both fluid balance and body weight assessments appears clinically important.

The current investigation was conducted in the ICU of a major tertiary referral hospital, which relies on an excellent level of experience of nursing staff and the availability of beds with built-in weighing scales. Nursing staff were trained on how to operate bed systems. Inaccuracies in both fluid balance and body weight estimations can be assumed to have occurred due to pragmatic nursing constraints amplified by highly imprecise indiscernible fluid losses formulas $[9,11,12,14]$. 
There are speculations about an attenuated influence of fluid accumulation over body weight in critically ill patients who are hospitalized for longer than five days, since muscle and fat loss as well as bone demineralization must play a major role after such a period [13-15]. Nevertheless, our results are still consistent with other cohorts of very short ICU hospitalizations $[8,13]$. The lack of a significant difference in the first 24-hour fluid balance between survivors and non-survivors, and even the modest absolute difference on corresponding body-weight change between them, might also reflect novel practices of careful fluid ressuscitation [21,22].

\section{CONCLUSION}

Obtaining daily fluid balance and body weight in ICU patients is complex and time-consuming. The assumption that a positive or negative fluid balance represents a similar gain or loss in body weight cannot be relied upon for estimating body-fluid status. There is little reason to believe that intense clinical teaching, procedure implementation, or any other intervention would enable daily fluid balance and body weight changes to be used reciprocally. Therefore, patient care and clinical decision-making should be based on other, more objective techniques.

We question the clinical importance of changes in fluid balance or body weight, as the volume load of specific compartments, such as intravascular volume, cardiac preload, extravascular lung water, is probably more relevant. Further research may assist in refining the value of routine fluid balance charting and of equipping ICUs with weighting beds.

\section{- CONCLICT OF INTEREST}

None to declare.

\section{- REFERENCES}

1. Dos Santos TOC, Oliveira MAS, Monte JCM, Batista MC, Pereira Junior VG, Dos Santos BFC, et al. Outcomes from a cohort of patients with acute kidney injury subjected to continuous venovenous hemodiafiltration: The role of negative fluid balance. PLoS One. 2017;12(4):e0175897.

2. Acheampong $A$, Vincent $J \mathrm{~L}$. A positive fluid balance is an independent prognostic factor in patients with sepsis. Crit Care. 2015;19:251.

3. de Oliveira FS, Freitas FG, Ferreira EM, de Castro I, Bafi AT, de Azevedo LC, et al. Positive fluid balance as a prognostic factor for mortality and acute kidney injury in severe sepsis and septic shock. J Crit Care. 2015;30(1):97-101.

4. Brotfain E, Koyfman L, Toledano R, Borer A, Fucs L, Galante O, et al. Positive fluid balance as a major predictor of clinical outcome of patients with sepsis/septic shock after ICU discharge. Am J Emerg Med. 2016;34(11):2122-6.

5. Sirvent JM, Ferri C, Baró A, Murcia C, Lorencio C. Fluid balance in sepsis and septic shock as a determining factor of mortality. Am J Emerg Med. 2015;33(2):186-9.

6. Huang $\mathrm{AC}$, Lee $\mathrm{TY}$, Ko MC, Huang $\mathrm{CH}$, Wang TY, Lin TY, et al. Fluid balance correlates with clinical course of multiple organ dysfunction syndrome and mortality in patients with septic shock. PLoS One. 2019;14(12):e0225423.

7. Shim HJ, Jang JY, Lee SH, Lee JG. The effect of positive balance on the outcomes of critically ill noncardiac postsurgical patients: a retrospective cohort study. J Crit Care. 2014;29(1):43-8.

8. You JW, Lee SJ, Kim YE, Cho YJ, Jeong YY, Kim HC, et al. Association between weight change and clinical outcomes in critically ill patients. J Crit Care. 2013;28(6):923-7.

9. Davies H, Leslie G, Jacob E, Morgan D. Estimation of Body Fluid Status by Fluid Balance and Body Weight in Critically III Adult Patients: A Systematic Review. Worldviews Evid Based Nurs. 2019;16(6):470-7.

10. Perren A, Markmann M, Merlani G, Marone C, Merlani P. Fluid balance in critically ill patients. Should we really rely on it? Minerva Anestesiol. 2011;77(8):802-11.

11. Cox P. Indiscernible water loss and its assessment in adult patients: a review. Acta Anaesthesiol Scand. 1987;31(8):771-6.

12. Pflaum SS. Investigation of intake-output as a means of assessing body fluid balance. Heart Lung. 1979;8(3):495-8.

13. Schneider AG, Baldwin I, Freitag E, Glassford N, Bellomo R. Estimation of fluid status changes in critically ill patients: fluid balance chart or electronic bed weight? J Crit Care. 2012;27(6):745.e7-12.

14. Schneider AG, Thorpe C, Dellbridge K, Matalanis G, Bellomo R. Electronic bed weighing vs daily fluid balance changes after cardiac surgery. J Crit Care. 2013;28(6):1113.e1-5.

15. Eastwood GM. Evaluating the reliability of recorded fluid balance to approximate body weight change in patients undergoing cardiac surgery. Heart Lung. 2006;35(1):27-33.

16. Köster M, Dennhardt S, Jüttner F, Hopf HB. Cumulative changes in weight but not fluid volume balances reflect fluid accumulation in ICU patients. Acta Anaesthesiol Scand. 2017;61(2):205-15.

17. Lodeserto F, Chohan H, Kasa KD, Pancoast TC. Accuracy of daily weights and fluid balance in medical intensive care unit patients with the intervention of electronic medical records and electronic bedside weight measurements. Chest. 2009;136(4).

18. Hill-rom. Total care bed system User Manual [Available from: https://monetmedical.com/wp-content/uploads/2017/11/HillRom-TotalCare-Service-Manual.pdf.

19. Al-Jubour M, Wilkinson B, Baldawi M, Watson J, Saraswat A, Galusca D. 1503: Association between Fluid Balance, Daily 
Available online at: www.jccm.ro

Weight Measurements and Surgical Intensive Care Outcomes. Crit Care Med. 2016;44(12):451.

20. Giavarina D. Understanding Bland Altman analysis. Biochem Med (Zagreb). 2015;25(2):141-51.

21. Mouncey PR, Osborn TM, Power GS, Harrison DA, Sadique MZ,
The Journal of Critical Care Medicine 2021;7(1) • 53

Grieve RD, et al. Trial of early, goal-directed resuscitation for septic shock. N Engl J Med. 2015;372(14):1301-11.

22. Peake SL, Delaney A, Bailey M, Bellomo R, Cameron PA, Cooper $D J$, et al. Goal-directed resuscitation for patients with early septic shock. N Engl J Med. 2014;371(16):1496-506. 